\title{
Highly Emissive Carbon Dots/Organosilicon Composites for Efficient and Stable Luminescent Solar Concentrators
}

Yuhan Wu ${ }^{\text {a }}$, Yuan Zhan a, ${ }^{\text {* }}$, Wei Xin ${ }^{\text {a }}$, Wenbing Cao ${ }^{\text {a }}$, Jinhua Li ${ }^{\text {a }}$, Ming Chen ${ }^{\text {b }, ~ X u a n f e n g ~ J i a n g ~}$

$$
\text { a, Jianying Wang a, *, Zhengguang Sun a, * }
$$

${ }^{a}$ Ministry-of-Education Key Laboratory for the Green Preparation and Application of Functional Materials, Hubei Key Laboratory of Polymer Materials, School of Materials Science and Engineering, Hubei University, Wuhan 430062, China

${ }^{\mathrm{b}}$ College of Chemistry and Materials Science, Jinan University, Guangzhou 510632, China

E-mail address: zy@hubu.edu.cn (Y. Zhan); wangjy_2002@163.com (J.Y. Wang); sunshine@ hubu.edu.cn (Z.G. Sun). 


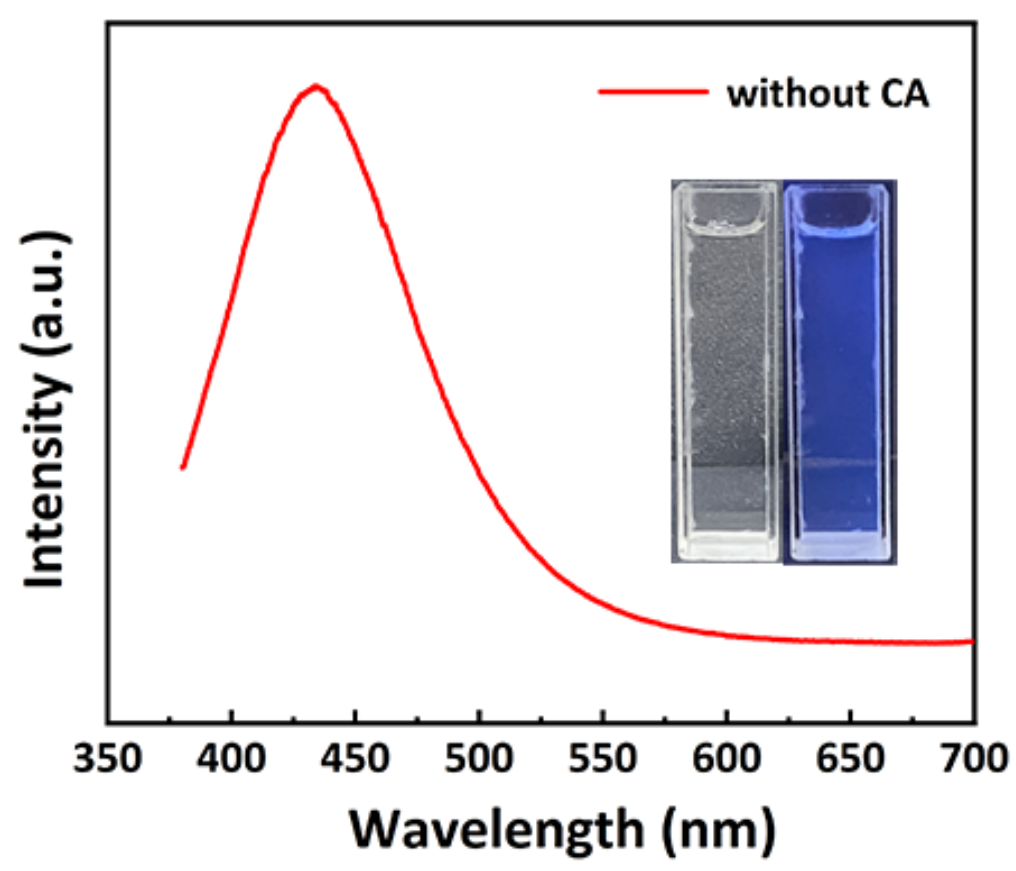

Fig. S1. PL spectrum of the solution without CA. The excitation wavelength is $360 \mathrm{~nm}$. The inset is the optical photograph of the solution under natural light and $365 \mathrm{~nm}$ UV light.

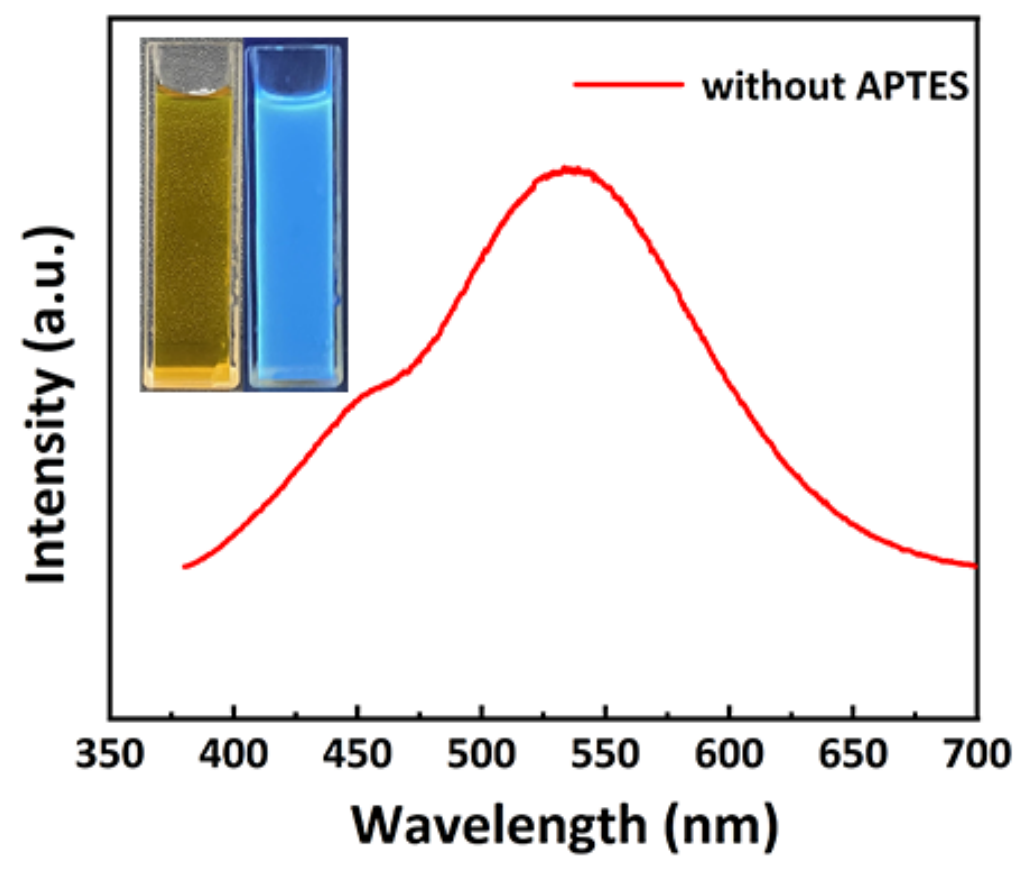

Fig. S2. PL spectrum of the solution without APTES. The excitation wavelength is $360 \mathrm{~nm}$. The inset is the optical photograph of the solution under natural light and $365 \mathrm{~nm}$ UV light. 


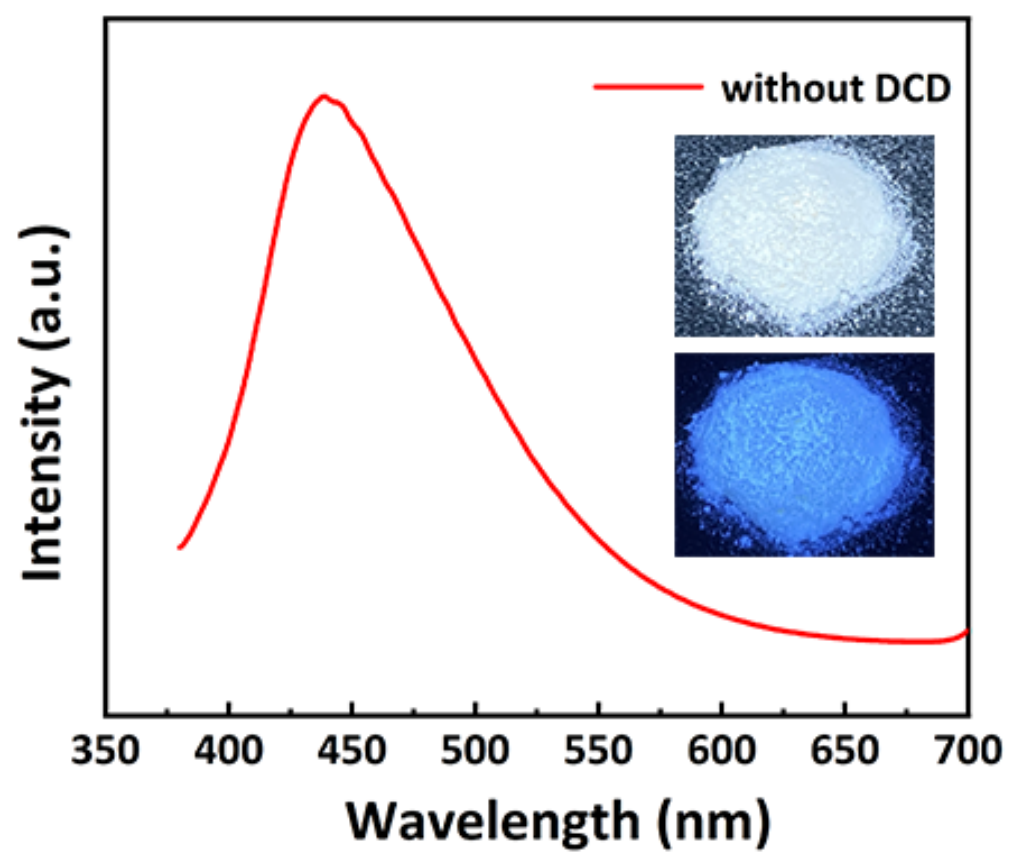

Fig. S3. PL spectrum of the solid powder without DCD. The excitation wavelength is $360 \mathrm{~nm}$. The inset is the optical photograph of the powder under natural light and $365 \mathrm{~nm}$ UV light.

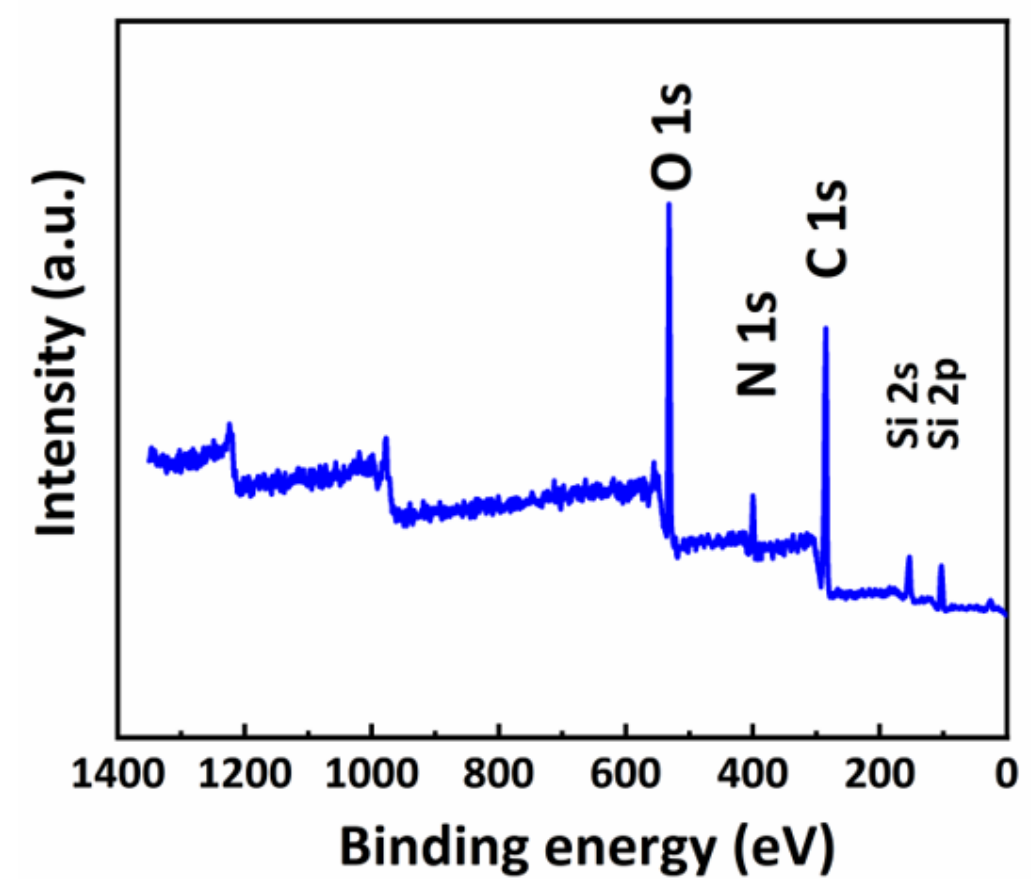

Fig. S4. Full survey of XPS spectrum for the OSi-CDs. 

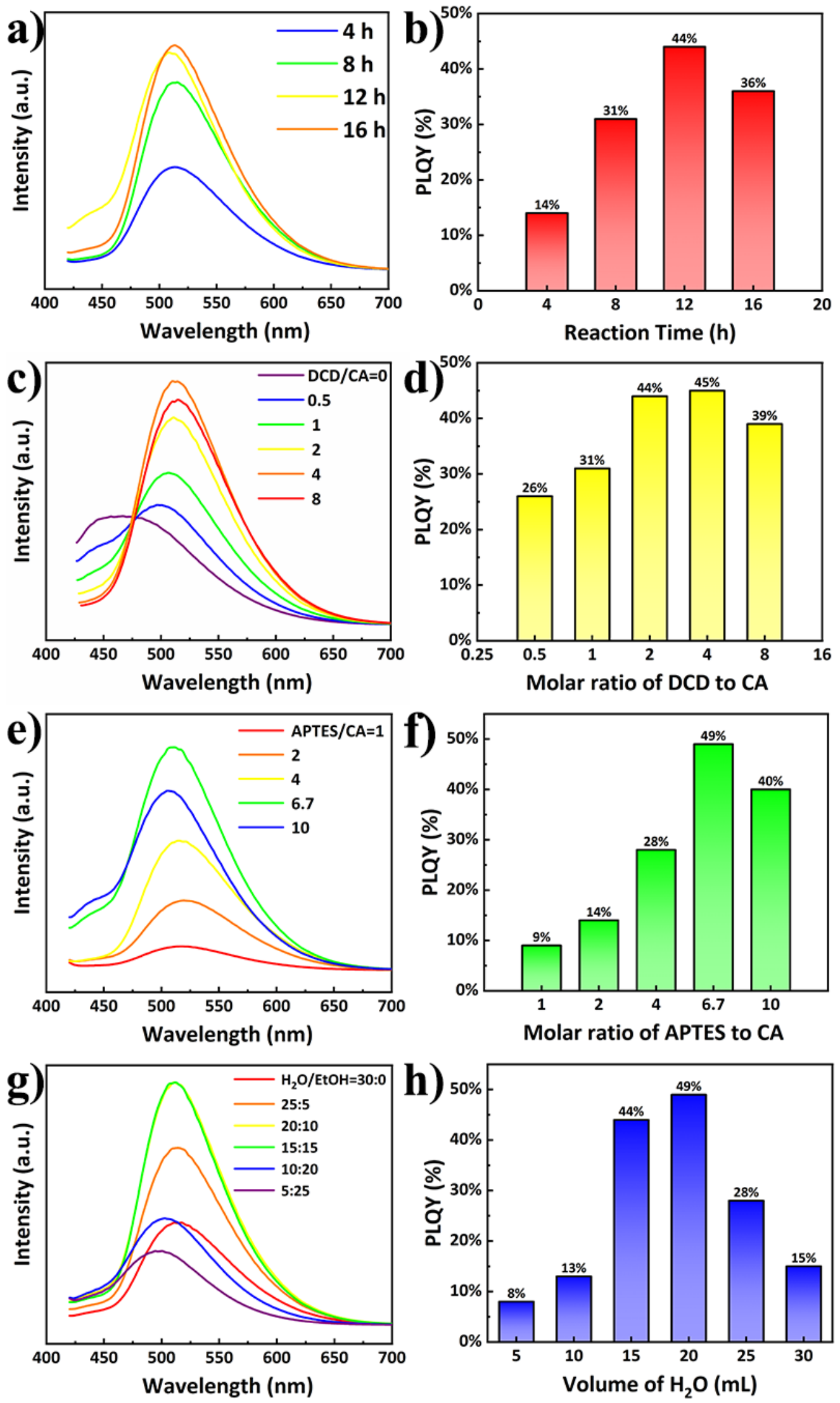

Fig. S5. PL spectra and corresponding PLQYs of the OSi-CDs synthesized at different $(a, b)$ reaction time, $(\mathrm{c}, \mathrm{d})$ molar ratio of DCD to $\mathrm{CA},(\mathrm{e}, \mathrm{f})$ molar ratio of APTES to CA, $(\mathrm{g}, \mathrm{h})$ volume ratio of $\mathrm{H}_{2} \mathrm{O}$ to $\mathrm{EtOH}$. 


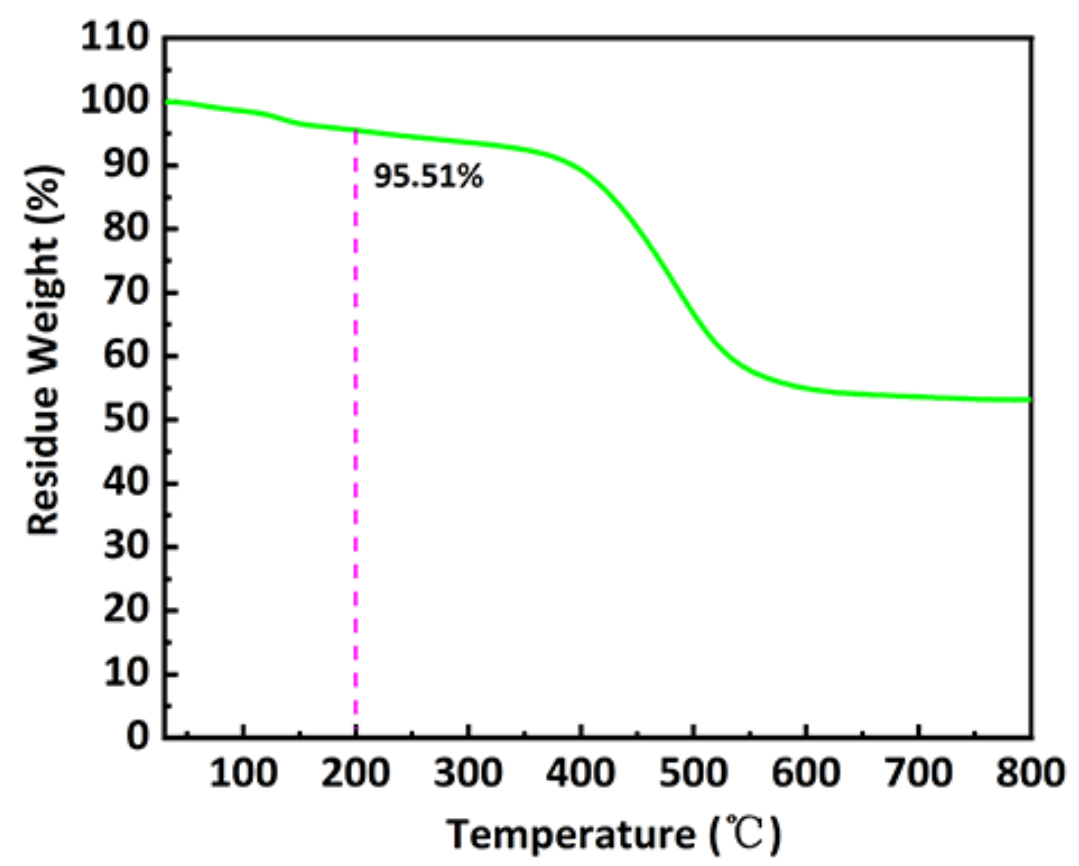

Fig. S6. TGA analysis of the OSi-CDs.

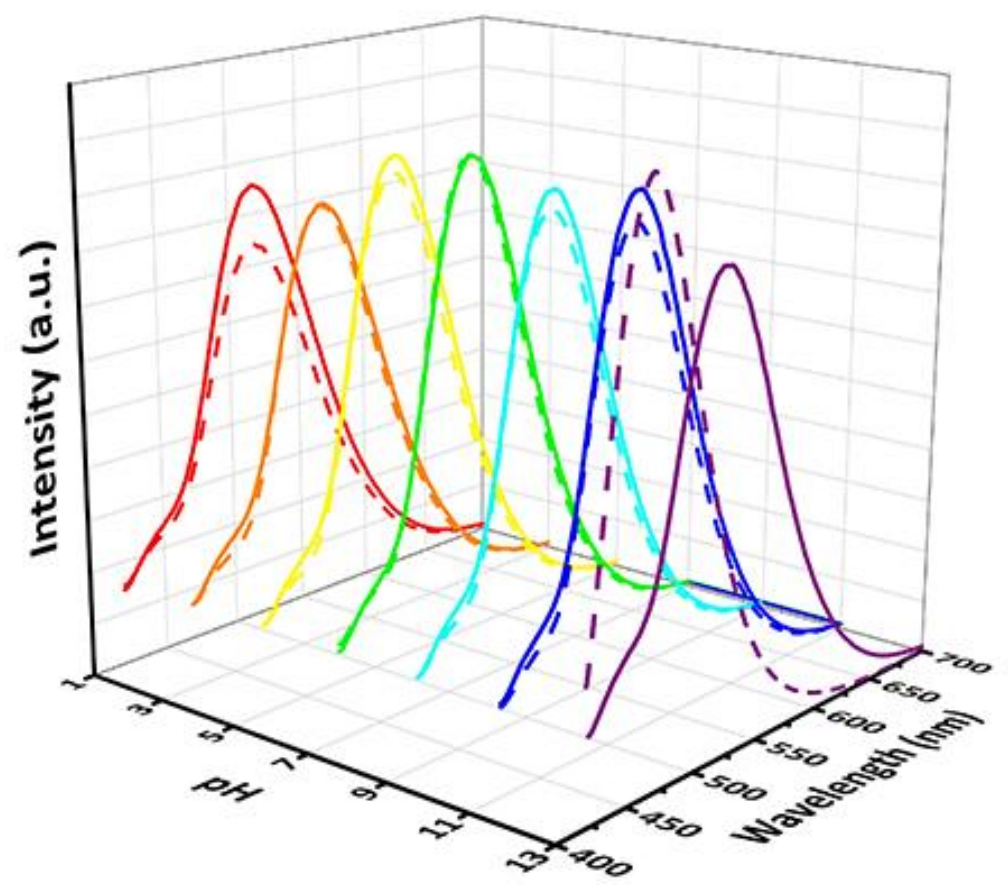

Fig. S7. PL spectra of the OSi-CDs in solutions with different $\mathrm{pH}$ before (solid line) and after (dash line) 7 days. The excitation wavelength is $400 \mathrm{~nm}$ 

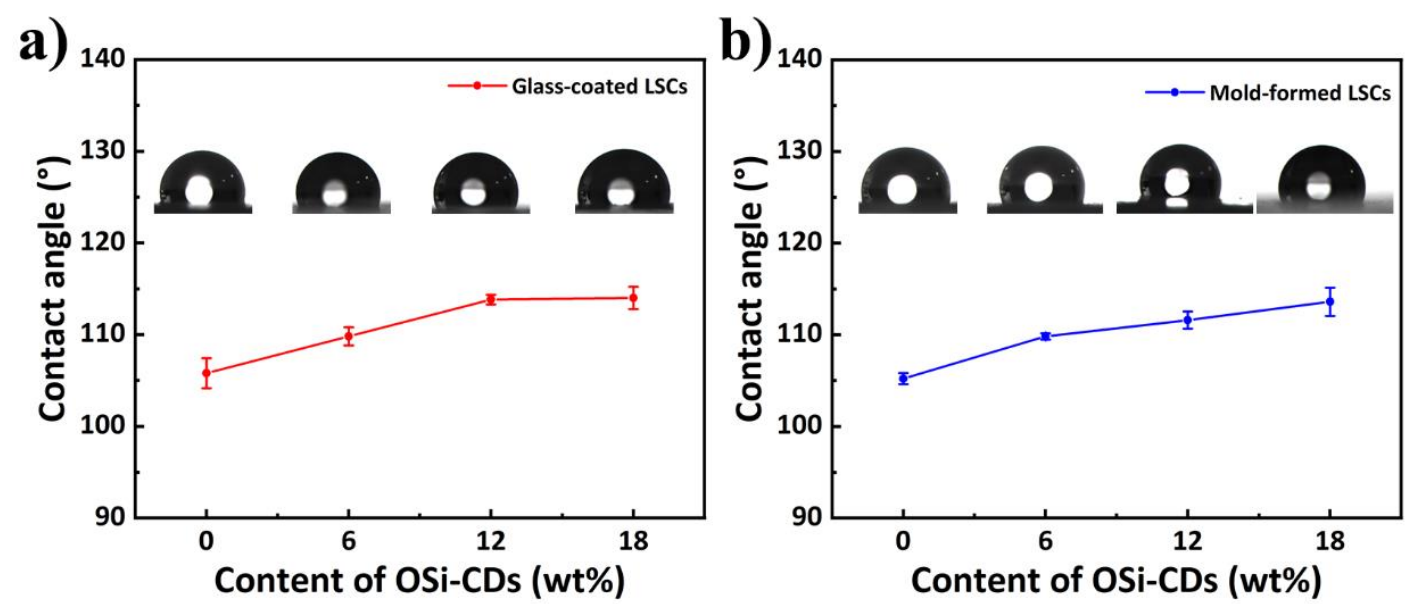

Fig. S8. Water contact angle of (a) the glass-coated LSCs and (b) mold-formed LSCs.
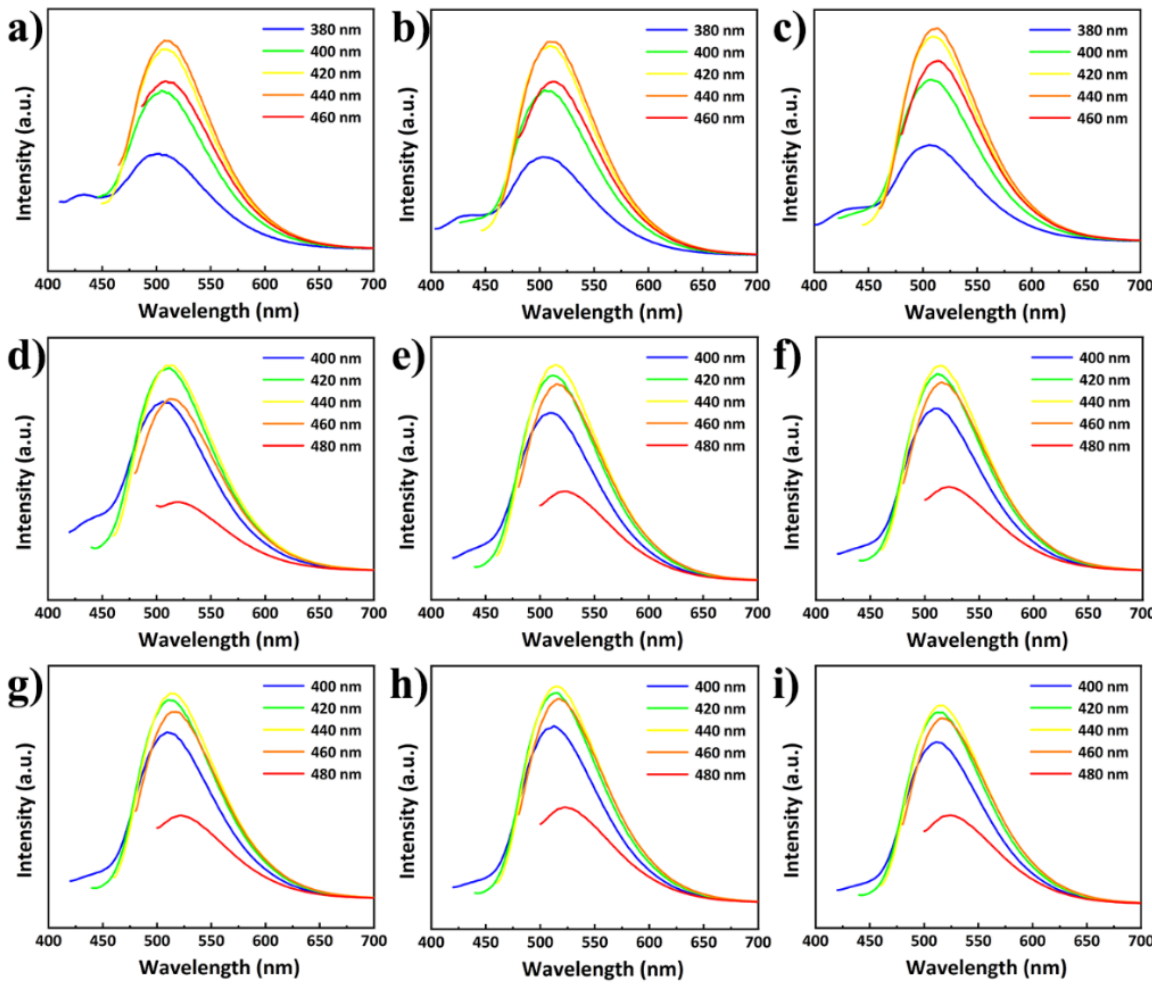

Fig. S9. PL spectra of the glass-coated LSCs with different content of OSi-CDs from $0.5-18 \mathrm{wt} \%$ at different excitation wavelengths. 

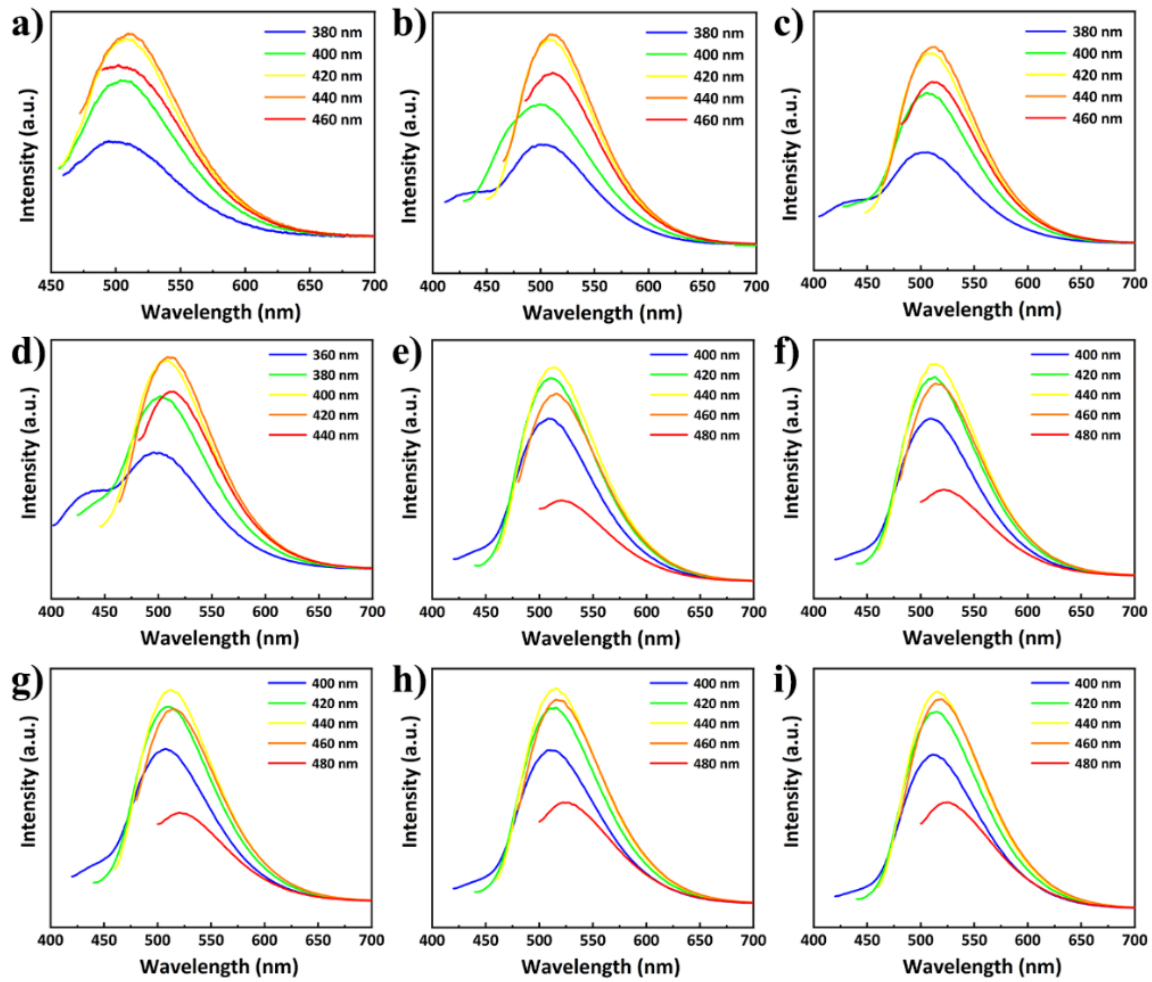

Fig. S10. PL spectra of the mold-formed LSCs with different content of OSi-CDs from $0.5-18 w t \%$ at different excitation wavelengths.
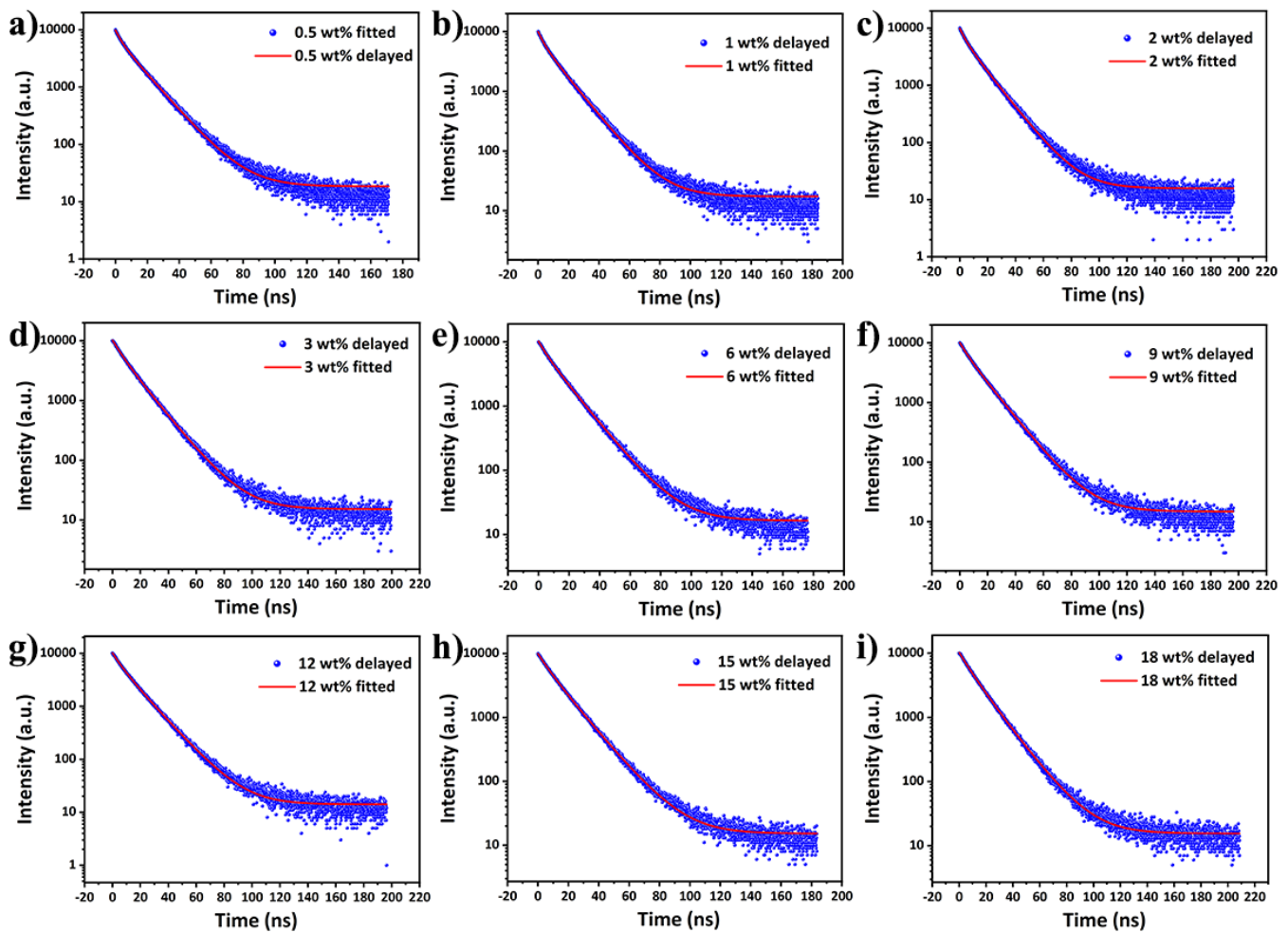

Fig. S11. Fluorescence decay curves of the glass-coated LSCs with different content of OSi-CDs from $0.5-18 \mathrm{wt} \%$. 

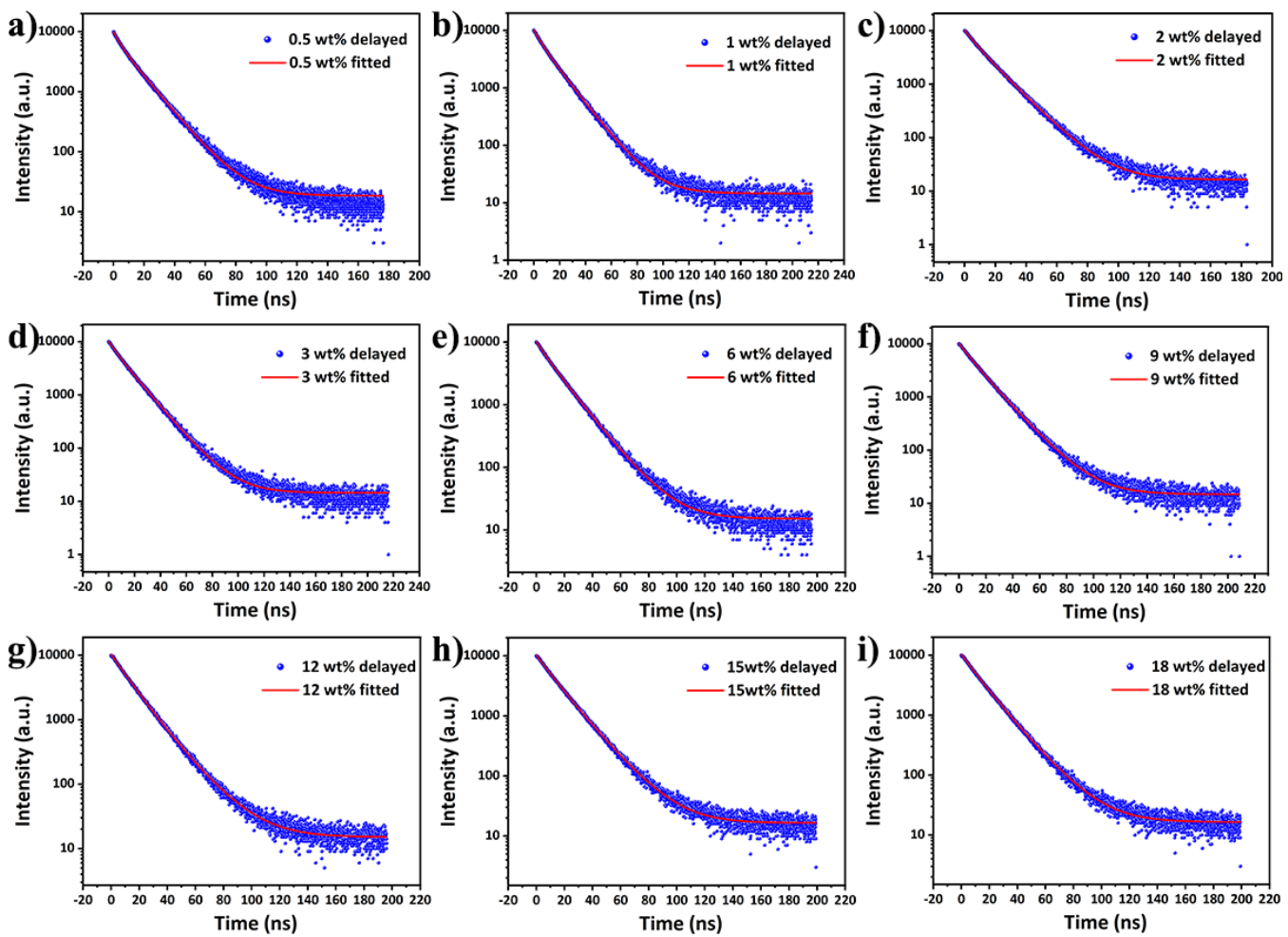

Fig. S12. Fluorescence decay curves of the mold-formed LSCs with different content of OSi-CDs from $0.5-18 w t \%$.
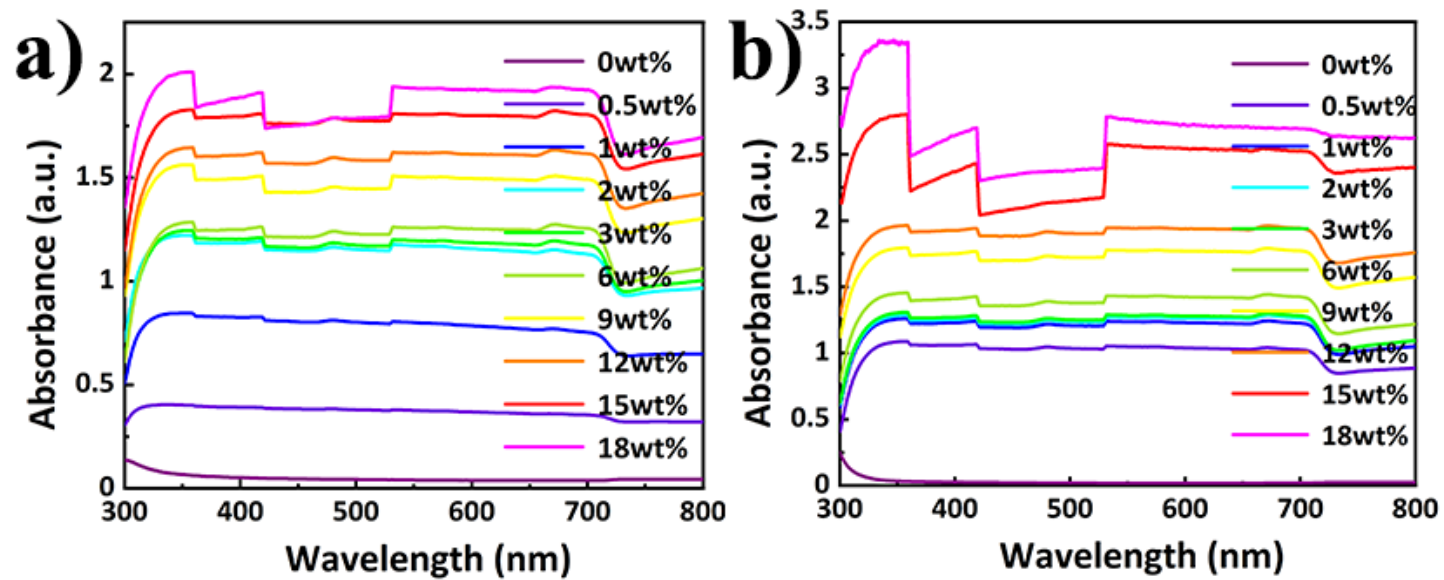

Fig. S13. UV-vis absorption spectra of (a) the glass-coated LSCs and (b) the mold-formed LSCs with different content of OSi-CDs from $0-18 \mathrm{wt} \%$. 


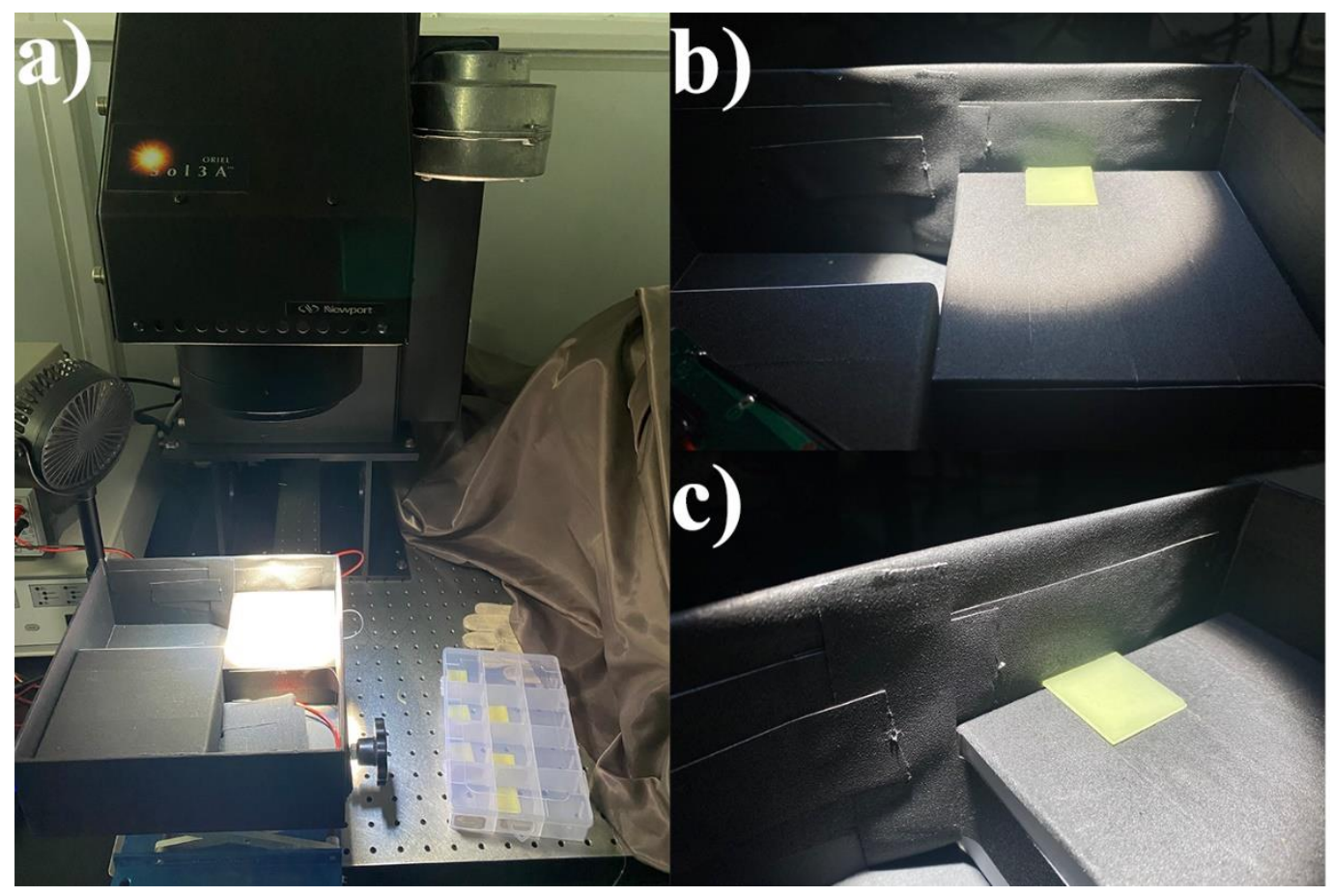

Fig. S14. Photographs of the produced LSCs under AM 1.5G radiation.
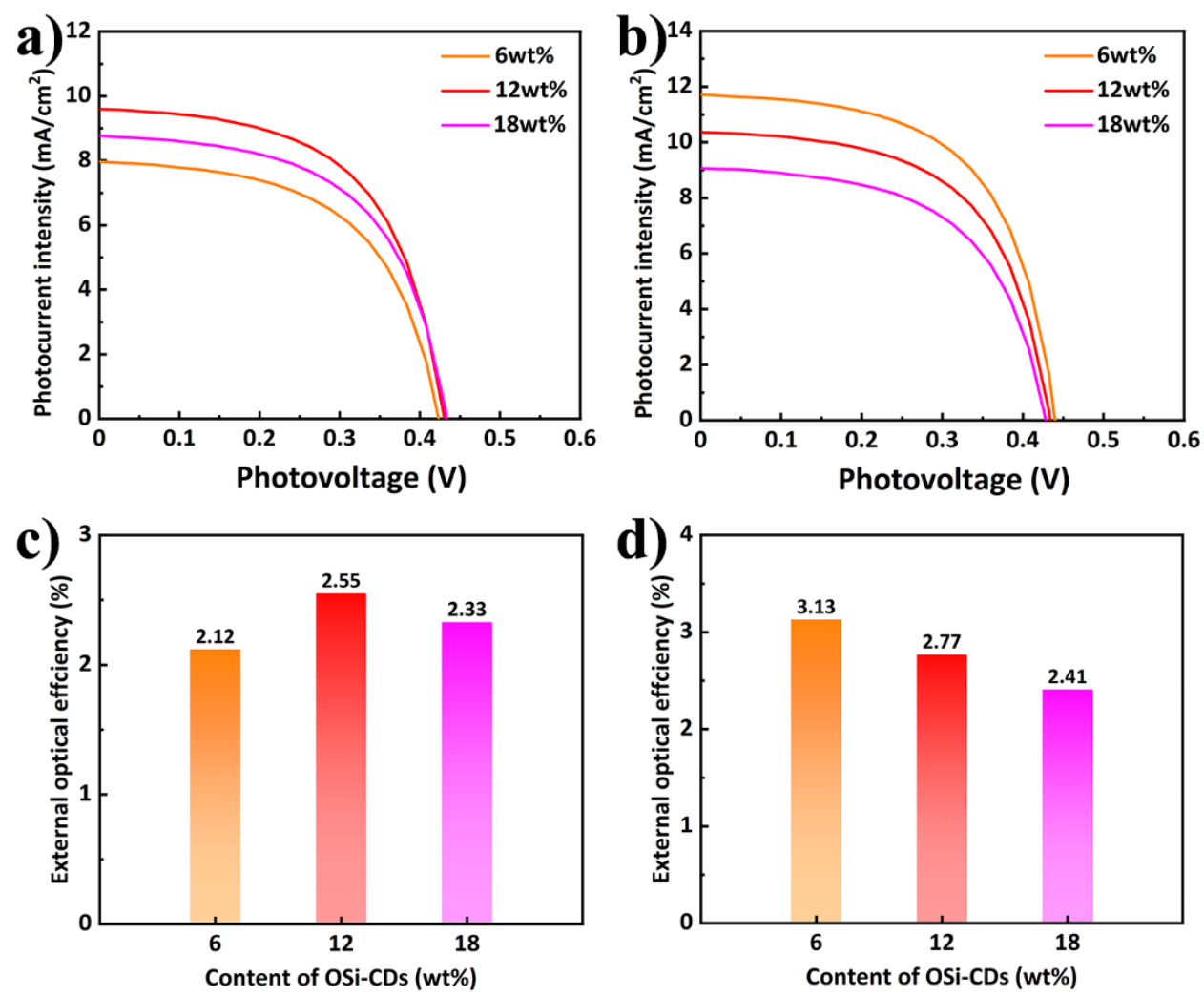

Fig. S15. Relationship of the photocurrent density and photovoltage $(J-V)$ of (a) the glass-coated LSCs $(50 \times 50 \times 1 \mathrm{~mm})$ and (b) the mold-formed LSCs $(50 \times 50 \times 1 \mathrm{~mm})$. The corresponding external optical efficiency histogram of (c) the glass-coated LSCs and (d) the mold-formed LSCs. 
Table S1. The preparation formula of the LSCs.

\begin{tabular}{cccc}
\hline Content of OSi-CDs & Component $\mathrm{A} / \mathrm{g}$ & Component B/g & OSi-CDs/g \\
\hline $0 \mathrm{wt} \%$ & 1.000 & 1.000 & 0.000 \\
$0.5 \mathrm{wt} \%$ & 1.000 & 1.000 & 0.010 \\
$1 \mathrm{wt} \%$ & 1.000 & 1.000 & 0.020 \\
$2 \mathrm{wt} \%$ & 1.000 & 1.000 & 0.041 \\
$3 \mathrm{wt} \%$ & 1.000 & 1.000 & 0.062 \\
$6 \mathrm{wt} \%$ & 1.000 & 1.000 & 0.128 \\
$9 \mathrm{wt} \%$ & 1.000 & 1.000 & 0.198 \\
$12 \mathrm{wt} \%$ & 1.000 & 1.000 & 0.273 \\
$15 \mathrm{wt} \%$ & 1.000 & 1.000 & 0.353 \\
$18 \mathrm{wt} \%$ & 1.000 & 1.000 & 0.439 \\
\hline
\end{tabular}

Table S2. Comparison of CDs-based composites with similar systems.

\begin{tabular}{cccccc}
\hline & Reaction time/h & Ex/nm & Em/nm & PLQY/\% & Ref \\
\hline SiCDs & 12 & 450 & 525 & 11 & 1 \\
$\mathrm{Si} / \mathrm{CDs}$ & 8 & 400 & 513 & 34.06 & 2 \\
$\mathrm{CNDs} /$ silica & 12 & 405 & 541 & 51 & 3 \\
$\mathrm{CDs} /$ silica & 12 & 400 & 498 & 50.3 & 4 \\
OSi-CDs & 12 & 400 & 517 & 49 & This work \\
\hline
\end{tabular}

Table S3. PL lifetime and PLQYs data of the glass-coated LSCs and OSi-CDs.

\begin{tabular}{cccccccc}
\hline $\begin{array}{c}\text { Content of } \\
\text { OSi-CDs }\end{array}$ & $\mathrm{A}_{1}$ & $\tau_{1} / \mathrm{ns}$ & $\mathrm{A}_{2}$ & $\tau_{2} / \mathrm{ns}$ & $\tau_{\text {avg } / \mathrm{ns}}$ & $\mathrm{R}^{2}$ & \multicolumn{2}{c}{ PLQYs } \\
\hline $0.5 \mathrm{wt} \%$ & 2685.97 & 3.54 & 7151.68 & 13.78 & 10.98 & 0.99970 & 21 \\
$1 \mathrm{wt} \%$ & 2756.01 & 3.58 & 7147.12 & 13.76 & 10.93 & 0.99970 & 25 \\
$2 \mathrm{wt} \%$ & 2682.24 & 4.11 & 7128.67 & 14.01 & 11.30 & 0.99970 & 26 \\
$3 \mathrm{wt} \%$ & 2668.15 & 6.57 & 7500.38 & 15.25 & 12.97 & 0.99968 & 37 \\
$6 \mathrm{wt} \%$ & 2375.25 & 5.75 & 7708.71 & 15.00 & 12.82 & 0.99967 & 35 \\
$9 \mathrm{wt} \%$ & 2244.23 & 5.84 & 7765.38 & 15.14 & 13.05 & 0.99971 & 35 \\
$12 \mathrm{wt} \%$ & 2752.56 & 5.92 & 7363.87 & 15.14 & 12.63 & 0.99973 & 33 \\
$15 \mathrm{wt} \%$ & 2442.16 & 7.14 & 7439.03 & 15.61 & 13.52 & 0.99972 & 32 \\
$18 \mathrm{wt} \%$ & 3033.44 & 8.53 & 7144.48 & 16.18 & 13.90 & 0.99972 & 30 \\
OSi-CDs & 1785.14 & 5.76 & 8014.11 & 15.38 & 13.63 & 0.99980 & 49 \\
\hline
\end{tabular}


Table S4. PL lifetime and PLQYs data of the mold-formed LSCs.

\begin{tabular}{cccccccc}
\hline $\begin{array}{c}\text { Content of } \\
\text { OSi-CDs }\end{array}$ & $\mathrm{A}_{1}$ & $\tau_{1} / \mathrm{ns}$ & $\mathrm{A}_{2}$ & $\tau_{2} / \mathrm{ns}$ & $\tau_{\mathrm{avg} / \mathrm{ns}}$ & $\mathrm{R}^{2}$ & \multicolumn{2}{c}{ PLQYs } \\
\hline $0.5 \mathrm{wt} \%$ & 2544.96 & 4.19 & 7359.82 & 14.24 & 11.66 & 0.99972 & 30 \\
$1 \mathrm{wt} \%$ & 2542.65 & 6.11 & 7473.10 & 15.16 & 12.86 & 0.99974 & 37 \\
$2 \mathrm{wt} \%$ & 2353.95 & 6.76 & 7646.80 & 15.44 & 13.40 & 0.99973 & 39 \\
$3 \mathrm{wt} \%$ & 2090.01 & 6.87 & 7919.87 & 15.44 & 13.65 & 0.99970 & 41 \\
$6 \mathrm{wt} \%$ & 2403.60 & 7.84 & 7670.90 & 15.90 & 13.98 & 0.99971 & 34 \\
$9 \mathrm{wt} \%$ & 3574.79 & 9.85 & 6486.01 & 16.75 & 14.30 & 0.99972 & 32 \\
$12 \mathrm{wt} \%$ & 6921.86 & 12.42 & 3270.73 & 19.32 & 14.63 & 0.99961 & 31 \\
$15 \mathrm{wt} \%$ & 4420.50 & 10.98 & 5785.82 & 17.39 & 14.61 & 0.99963 & 31 \\
$18 \mathrm{wt} \%$ & 3005.92 & 11.47 & 6992.17 & 16.35 & 14.88 & 0.99972 & 29 \\
\hline
\end{tabular}

Table S5. Photovoltaic parameters of the glass-coated LSCs $(25 \times 25 \times 1 \mathrm{~mm})$ with different content of OSi-CDs before being kept in $100{ }^{\circ} \mathrm{C}$ for $168 \mathrm{~h}$.

\begin{tabular}{ccccccc}
\hline & $J_{s c}\left(\mathrm{~mA} / \mathrm{cm}^{2}\right)$ & $V_{o c}(\mathrm{~V})$ & $F F(\%)$ & $G$ & $\eta_{o p t}(\%)$ & PCE $(\%)$ \\
\hline $0 \mathrm{wt} \%$ & 1.67 & 0.31 & 44.43 & 6.25 & 0.65 & 0.009 \\
$0.5 \mathrm{wt} \%$ & 3.53 & 0.37 & 49.00 & 6.25 & 1.37 & 0.026 \\
$1 \mathrm{wt} \%$ & 4.70 & 0.39 & 51.28 & 6.25 & 1.82 & 0.038 \\
$2 \mathrm{wt} \%$ & 6.08 & 0.40 & 54.28 & 6.25 & 2.36 & 0.053 \\
$3 \mathrm{wt} \%$ & 6.36 & 0.41 & 53.69 & 6.25 & 2.47 & 0.056 \\
$6 \mathrm{wt} \%$ & 8.80 & 0.43 & 55.50 & 6.25 & 3.41 & 0.084 \\
$9 \mathrm{wt} \%$ & 10.87 & 0.43 & 57.98 & 6.25 & 4.21 & 0.108 \\
$12 \mathrm{wt} \%$ & 11.62 & 0.43 & 58.64 & 6.25 & 4.50 & 0.117 \\
$15 \mathrm{wt} \%$ & 11.24 & 0.45 & 57.53 & 6.25 & 4.36 & 0.116 \\
$18 \mathrm{wt} \%$ & 11.15 & 0.44 & 57.48 & 6.25 & 4.32 & 0.113 \\
\hline
\end{tabular}


Table S6. Photovoltaic parameters of the mold-formed LSCs $(25 \times 25 \times 1 \mathrm{~mm})$ with different content of OSi-CDs before being kept in $100{ }^{\circ} \mathrm{C}$ for $168 \mathrm{~h}$.

\begin{tabular}{ccccccc}
\hline & $J_{s c}\left(\mathrm{~mA} / \mathrm{cm}^{2}\right)$ & $V_{o c}(\mathrm{~V})$ & $F F(\%)$ & $G$ & $\eta_{o p t}(\%)$ & PCE $(\%)$ \\
\hline $0 \mathrm{wt} \%$ & 2.35 & 0.35 & 46.31 & 6.25 & 0.91 & 0.015 \\
$0.5 \mathrm{wt} \%$ & 10.19 & 0.42 & 56.78 & 6.25 & 3.95 & 0.097 \\
$1 \mathrm{wt} \%$ & 12.66 & 0.43 & 59.15 & 6.25 & 4.91 & 0.129 \\
$2 \mathrm{wt} \%$ & 14.79 & 0.44 & 60.08 & 6.25 & 5.73 & 0.156 \\
$3 \mathrm{wt} \%$ & 15.19 & 0.44 & 59.70 & 6.25 & 5.89 & 0.160 \\
6wt\% & 14.42 & 0.44 & 58.47 & 6.25 & 5.59 & 0.148 \\
9wt\% & 13.90 & 0.44 & 58.21 & 6.25 & 5.39 & 0.142 \\
$12 \mathrm{wt} \%$ & 13.12 & 0.43 & 58.85 & 6.25 & 5.09 & 0.133 \\
$15 \mathrm{wt} \%$ & 12.73 & 0.43 & 59.01 & 6.25 & 4.93 & 0.129 \\
$18 \mathrm{wt} \%$ & 12.23 & 0.43 & 58.76 & 6.25 & 4.74 & 0.124 \\
\hline
\end{tabular}

Table S7. Photovoltaic parameters of the glass-coated LSCs $(50 \times 50 \times 1 \mathrm{~mm})$ with different content of OSi-CDs.

\begin{tabular}{ccccccc}
\hline & $J_{s c}\left(\mathrm{~mA} / \mathrm{cm}^{2}\right)$ & $V_{o c}(\mathrm{~V})$ & $F F(\%)$ & $G$ & $\eta_{\text {opt }}(\%)$ & PCE $(\%)$ \\
\hline $6 \mathrm{wt} \%$ & 7.95 & 0.42 & 56.60 & 12.5 & 2.12 & 0.038 \\
$12 \mathrm{wt} \%$ & 9.58 & 0.43 & 57.53 & 12.5 & 2.55 & 0.047 \\
$18 \mathrm{wt} \%$ & 8.73 & 0.43 & 57.54 & 12.5 & 2.33 & 0.043 \\
\hline
\end{tabular}

Table S8. Photovoltaic parameters of the mold-formed LSCs $(50 \times 50 \times 1 \mathrm{~mm})$ with different content of OSi-CDs.

\begin{tabular}{ccccccc}
\hline & $J_{s c}\left(\mathrm{~mA} / \mathrm{cm}^{2}\right)$ & $V_{o c}(\mathrm{~V})$ & $F F(\%)$ & $G$ & $\eta_{o p t}(\%)$ & PCE $(\%)$ \\
\hline $6 \mathrm{wt} \%$ & 11.74 & 0.44 & 58.66 & 12.5 & 3.13 & 0.061 \\
$12 \mathrm{wt} \%$ & 10.38 & 0.43 & 58.48 & 12.5 & 2.77 & 0.052 \\
$18 \mathrm{wt} \%$ & 9.02 & 0.43 & 56.72 & 12.5 & 2.41 & 0.044 \\
\hline
\end{tabular}


Table S9. Photovoltaic parameters of the glass-coated LSCs with different content of OSi-CDs after being kept in $100^{\circ} \mathrm{C}$ for $168 \mathrm{~h}$.

\begin{tabular}{ccccccc}
\hline & $J_{s c}\left(\mathrm{~mA} / \mathrm{cm}^{2}\right)$ & $V_{o c}(\mathrm{~V})$ & $F F(\%)$ & $G$ & $\eta_{o p t}(\%)$ & PCE $(\%)$ \\
\hline $0 \mathrm{wt} \%$ & 1.58 & 0.30 & 44.30 & 6.25 & 0.61 & 0.008 \\
$0.5 \mathrm{wt} \%$ & 3.31 & 0.37 & 48.99 & 6.25 & 1.28 & 0.024 \\
$1 \mathrm{wt} \%$ & 4.45 & 0.40 & 48.88 & 6.25 & 1.72 & 0.035 \\
$2 \mathrm{wt} \%$ & 5.66 & 0.43 & 48.89 & 6.25 & 2.19 & 0.048 \\
$3 \mathrm{wt} \%$ & 6.56 & 0.43 & 53.53 & 6.25 & 2.54 & 0.060 \\
$6 \mathrm{wt} \%$ & 8.28 & 0.44 & 55.72 & 6.25 & 3.21 & 0.081 \\
$9 \mathrm{wt} \%$ & 10.81 & 0.45 & 57.97 & 6.25 & 4.19 & 0.113 \\
$12 \mathrm{wt} \%$ & 11.34 & 0.45 & 58.20 & 6.25 & 4.40 & 0.119 \\
$15 \mathrm{wt} \%$ & 11.01 & 0.43 & 58.30 & 6.25 & 4.27 & 0.110 \\
$18 \mathrm{wt} \%$ & 10.99 & 0.43 & 57.98 & 6.25 & 4.26 & 0.110 \\
\hline
\end{tabular}

Table S10. Photovoltaic parameters of the mold-formed LSCs with different content of OSi-CDs after being kept in $100^{\circ} \mathrm{C}$ for $168 \mathrm{~h}$.

\begin{tabular}{ccccccc}
\hline & $J_{s c}\left(\mathrm{~mA} / \mathrm{cm}^{2}\right)$ & $V_{o c}(\mathrm{~V})$ & $F F(\%)$ & $G$ & $\eta_{\text {opt }}(\%)$ & PCE $(\%)$ \\
\hline $0 \mathrm{wt} \%$ & 2.36 & 0.35 & 44.79 & 6.25 & 0.91 & 0.015 \\
$0.5 \mathrm{wt} \%$ & 9.60 & 0.41 & 57.67 & 6.25 & 3.72 & 0.091 \\
$1 \mathrm{wt} \%$ & 11.71 & 0.42 & 59.57 & 6.25 & 4.54 & 0.117 \\
$2 \mathrm{wt} \%$ & 13.11 & 0.43 & 59.78 & 6.25 & 5.08 & 0.135 \\
$3 \mathrm{wt} \%$ & 14.42 & 0.44 & 58.47 & 6.25 & 5.59 & 0.148 \\
$6 \mathrm{wt} \%$ & 14.18 & 0.44 & 58.66 & 6.25 & 5.50 & 0.146 \\
9wt\% & 13.46 & 0.44 & 58.08 & 6.25 & 5.22 & 0.138 \\
$12 \mathrm{wt} \%$ & 12.62 & 0.43 & 58.42 & 6.25 & 4.89 & 0.127 \\
$15 \mathrm{wt} \%$ & 12.55 & 0.43 & 58.74 & 6.25 & 4.86 & 0.127 \\
$18 \mathrm{wt} \%$ & 11.89 & 0.43 & 58.09 & 6.25 & 4.61 & 0.119 \\
\hline
\end{tabular}


Table S11. Photovoltaic parameters of the glass-coated LSCs with different content of OSi-CDs before $168 \mathrm{~h} \mathrm{UV}$ radiation.

\begin{tabular}{ccccccc}
\hline & $J_{s c}\left(\mathrm{~mA} / \mathrm{cm}^{2}\right)$ & $V_{o c}(\mathrm{~V})$ & $F F(\%)$ & $G$ & $\eta_{\text {opt }}(\%)$ & PCE $(\%)$ \\
\hline $0 \mathrm{wt} \%$ & 1.64 & 0.31 & 45.24 & 6.25 & 0.64 & 0.009 \\
$0.5 \mathrm{wt} \%$ & 3.61 & 0.38 & 48.84 & 6.25 & 1.40 & 0.027 \\
$1 \mathrm{wt} \%$ & 4.74 & 0.39 & 51.93 & 6.25 & 1.84 & 0.038 \\
$2 \mathrm{wt} \%$ & 6.07 & 0.40 & 54.78 & 6.25 & 2.35 & 0.053 \\
$3 \mathrm{wt} \%$ & 6.98 & 0.41 & 47.52 & 6.25 & 2.71 & 0.054 \\
$6 \mathrm{wt} \%$ & 8.68 & 0.43 & 55.73 & 6.25 & 3.36 & 0.083 \\
$9 \mathrm{wt} \%$ & 10.66 & 0.43 & 57.59 & 6.25 & 4.13 & 0.106 \\
$12 \mathrm{wt} \%$ & 11.59 & 0.44 & 57.26 & 6.25 & 4.49 & 0.117 \\
$15 \mathrm{wt} \%$ & 11.16 & 0.44 & 56.41 & 6.25 & 4.33 & 0.111 \\
$18 \mathrm{wt} \%$ & 11.12 & 0.44 & 57.23 & 6.25 & 4.31 & 0.112 \\
\hline
\end{tabular}

Table S12. Photovoltaic parameters of the mold-formed LSCs with different content of OSi-CDs before $168 \mathrm{~h} \mathrm{UV}$ radiation.

\begin{tabular}{ccccccc}
\hline & $J_{s c}\left(\mathrm{~mA} / \mathrm{cm}^{2}\right)$ & $V_{o c}(\mathrm{~V})$ & $F F(\%)$ & $G$ & $\eta_{o p t}(\%)$ & PCE (\%) \\
\hline $0 \mathrm{wt} \%$ & 2.33 & 0.34 & 46.71 & 6.25 & 0.90 & 0.015 \\
$0.5 \mathrm{wt} \%$ & 9.83 & 0.42 & 56.68 & 6.25 & 3.81 & 0.094 \\
$1 \mathrm{wt} \%$ & 12.20 & 0.43 & 54.14 & 6.25 & 4.73 & 0.114 \\
$2 \mathrm{wt} \%$ & 14.33 & 0.44 & 59.47 & 6.25 & 5.55 & 0.150 \\
$3 \mathrm{wt} \%$ & 14.96 & 0.44 & 59.40 & 6.25 & 5.80 & 0.156 \\
$6 \mathrm{wt} \%$ & 14.42 & 0.44 & 58.16 & 6.25 & 5.59 & 0.148 \\
$9 \mathrm{wt} \%$ & 13.82 & 0.44 & 58.05 & 6.25 & 5.36 & 0.141 \\
$12 \mathrm{wt} \%$ & 12.98 & 0.43 & 58.95 & 6.25 & 5.03 & 0.132 \\
$15 \mathrm{wt} \%$ & 12.69 & 0.43 & 58.83 & 6.25 & 4.92 & 0.128 \\
$18 \mathrm{wt} \%$ & 12.21 & 0.43 & 58.66 & 6.25 & 4.73 & 0.123 \\
\hline
\end{tabular}


Table S13. Photovoltaic parameters of the glass-coated LSCs with different content of OSi-CDs after $168 \mathrm{~h} \mathrm{UV}$ radiation.

\begin{tabular}{ccccccc}
\hline & $J_{s c}\left(\mathrm{~mA} / \mathrm{cm}^{2}\right)$ & $V_{o c}(\mathrm{~V})$ & $F F(\%)$ & $G$ & $\eta_{o p t}(\%)$ & PCE $(\%)$ \\
\hline $0 \mathrm{wt} \%$ & 1.57 & 0.30 & 44.59 & 6.25 & 0.61 & 0.008 \\
$0.5 \mathrm{wt} \%$ & 3.22 & 0.36 & 49.17 & 6.25 & 1.25 & 0.023 \\
$1 \mathrm{wt} \%$ & 4.29 & 0.38 & 51.53 & 6.25 & 1.66 & 0.034 \\
$2 \mathrm{wt} \%$ & 5.48 & 0.39 & 53.34 & 6.25 & 2.12 & 0.046 \\
$3 \mathrm{wt} \%$ & 5.68 & 0.39 & 51.46 & 6.25 & 2.20 & 0.046 \\
$6 \mathrm{wt} \%$ & 7.50 & 0.41 & 53.66 & 6.25 & 2.91 & 0.066 \\
$9 \mathrm{wt} \%$ & 9.73 & 0.43 & 54.73 & 6.25 & 3.77 & 0.092 \\
$12 \mathrm{wt} \%$ & 10.05 & 0.43 & 55.07 & 6.25 & 3.90 & 0.095 \\
$15 \mathrm{wt} \%$ & 9.48 & 0.42 & 55.51 & 6.25 & 3.67 & 0.088 \\
$18 \mathrm{wt} \%$ & 9.77 & 0.43 & 54.75 & 6.25 & 3.79 & 0.092 \\
\hline
\end{tabular}

Table S14. Photovoltaic parameters of the mold-formed LSCs with different content of OSi-CDs after $168 \mathrm{~h}$ UV radiation.

\begin{tabular}{ccccccc}
\hline & $J_{s c}\left(\mathrm{~mA} / \mathrm{cm}^{2}\right)$ & $V_{o c}(\mathrm{~V})$ & $F F(\%)$ & $G$ & $\eta_{\text {opt }}(\%)$ & PCE (\%) \\
\hline $0 \mathrm{wt} \%$ & 2.31 & 0.35 & 45.76 & 6.25 & 0.90 & 0.015 \\
$0.5 \mathrm{wt} \%$ & 9.47 & 0.41 & 56.92 & 6.25 & 3.67 & 0.088 \\
$1 \mathrm{wt} \%$ & 11.27 & 0.42 & 58.94 & 6.25 & 4.37 & 0.112 \\
$2 \mathrm{wt} \%$ & 13.05 & 0.43 & 59.34 & 6.25 & 5.06 & 0.133 \\
$3 \mathrm{wt} \%$ & 13.46 & 0.43 & 59.78 & 6.25 & 5.22 & 0.138 \\
$6 \mathrm{wt} \%$ & 12.23 & 0.43 & 58.38 & 6.25 & 4.74 & 0.123 \\
$9 \mathrm{wt} \%$ & 12.14 & 0.43 & 58.43 & 6.25 & 4.71 & 0.122 \\
$12 \mathrm{wt} \%$ & 12.02 & 0.43 & 58.24 & 6.25 & 4.66 & 0.120 \\
$15 \mathrm{wt} \%$ & 11.52 & 0.42 & 59.32 & 6.25 & 4.47 & 0.115 \\
$18 \mathrm{wt} \%$ & 10.56 & 0.42 & 57.72 & 6.25 & 4.09 & 0.102 \\
\hline
\end{tabular}


Table S15. The $\eta_{\text {opt }}$ and stability for LSCs with different luminophore and dimension.

\begin{tabular}{|c|c|c|c|c|c|c|c|}
\hline \multirow{2}{*}{$\begin{array}{c}\text { Type of } \\
\text { luminophore }\end{array}$} & \multirow{2}{*}{ Luminophore } & \multirow{2}{*}{$\begin{array}{l}\text { Dimensions } \\
\quad\left(\mathrm{cm}^{3}\right)\end{array}$} & \multirow{2}{*}{$\eta_{\text {opt }}$} & \multicolumn{3}{|c|}{ Stability } & \multirow{2}{*}{ Ref } \\
\hline & & & & Conditions & PL decline & $\eta_{\text {opt }}$ decline & \\
\hline \multirow[t]{2}{*}{ Perovskite NCs } & $\mathrm{CsPbI}_{3}$ & $2 \times 2 \times 0.2$ & $3.10 \%$ & Ambient conditions for 2 months & NA & $6.40 \%$ & 5 \\
\hline & & & & UV light $\left(100 \mathrm{~mW} / \mathrm{cm}^{2}\right)$ for $10 \mathrm{~h}$ & $10 \%$ & NA & \\
\hline Perovskite QDs & $\mathrm{CsPb}\left(\mathrm{Br}_{0.2} \mathrm{I}_{0.8}\right)_{3}$ & $9 \times 1.3 \times 0.2$ & $2.00 \%$ & Ambient conditions for 3 months & NA & $\begin{array}{c}\text { No significant } \\
\text { change }\end{array}$ & 6 \\
\hline Perovskites & $\mathrm{CH}_{3} \mathrm{NH}_{3} \mathrm{PbBr}_{3}$ & $5 \times 3 \times 0.5$ & $1.57 \%$ & UV light $(9 \mathrm{~W})$ for $12 \mathrm{~h}$ & $15 \%$ & NA & 7 \\
\hline \multirow{2}{*}{$\begin{array}{l}\text { CDs and } \\
\text { perovskite QDs } \\
\text { (tandem) }\end{array}$} & $\begin{array}{c}\mathrm{CDs} \\
\mathrm{CsPb}\left(\mathrm{I}_{\mathrm{x}} \mathrm{Br}_{1-\mathrm{x}}\right)_{3}\end{array}$ & \multirow[t]{2}{*}{$10 \times 10 \times 0.2$} & \multirow[t]{2}{*}{$3.05 \%$} & $\begin{array}{l}\text { UV light }\left(200 \mathrm{~mW} / \mathrm{cm}^{2}\right) \text { with a } \\
\text { humidity of } 40 \% \text { at } 25^{\circ} \mathrm{C} \text { for } 70 \mathrm{~h}\end{array}$ & $\begin{array}{l}\text { No significant } \\
\text { variation }\end{array}$ & NA & 8 \\
\hline & $\mathrm{CsPb}\left(\mathrm{Cl}_{\mathrm{x}} \mathrm{Br}_{1-\mathrm{x}}\right)_{3}$ & & & Ambient conditions for 6 months & NA & $\begin{array}{c}\text { No significant } \\
\text { change }\end{array}$ & \\
\hline \multirow[t]{2}{*}{ CDs } & \multirow{2}{*}{$\begin{array}{l}\text { OLA-modified } \\
\text { CDs }\end{array}$} & \multirow[t]{2}{*}{$10 \times 1.5 \times 0.2$} & \multirow[t]{2}{*}{$1.20 \%$} & $\begin{array}{l}\mathrm{UV} \text { light }\left(1.3 \mathrm{~W} / \mathrm{cm}^{2}\right) \text { at room } \\
\text { temperature (humidity of } \sim 40 \% \text { ) } \\
\text { for } 12 \mathrm{~h}\end{array}$ & No change & NA & 9 \\
\hline & & & & Ambient conditions for 4 months & NA & $\begin{array}{c}\text { No significant } \\
\text { change }\end{array}$ & \\
\hline CDs & $\mathrm{Si}-\mathrm{CDs}$ & $2.5 \times 2.5 \times 0.1$ & $7.58 \%$ & Ambient conditions for 2 months & NA & $9.40 \%$ & 1 \\
\hline \multirow[t]{2}{*}{ CDs } & \multirow[t]{2}{*}{ OSi-CDs } & \multirow[t]{2}{*}{$2.5 \times 2.5 \times 0.1$} & \multirow[t]{2}{*}{$5.89 \%$} & UV light $\left(1 \mathrm{~W} / \mathrm{cm}^{2}\right)$ for $168 \mathrm{~h}$ & $47 \%$ & $8.83 \%$ & This \\
\hline & & & & $100^{\circ} \mathrm{C}$ for $168 \mathrm{~h}$ & $11 \%$ & $5.00 \%$ & work \\
\hline
\end{tabular}

Note: "NA" stands for not available, indicating that no relevant test is given in the article. 
References

1. Wu, J.; Xin, W.; Wu, Y.; Zhan, Y.; Li, J.; Wang, J.; Huang, S.; Wang, X. Solid-State Photoluminescent Silicone-Carbon Dots/Dendrimer Composites for Highly Efficient Luminescent Solar Concentrators. Chem. Eng. J. 2021, 422, 130158.

2. Yan, F.; Zhang, H.; Xu, J.; Wu, Y.; Zang, Y.; Sun, J. Color Emission Carbon Dots with Quench-Resixastant Solid-State Fluorescence for Light-Emitting Diodes. ACS Sustain. Chem. Eng, 2021, 9, 3901-3908.

3. Qin, Z.; Wen, M.; Bai, J.; Cui, J.; Miao, R.; Zhang, X.; Zhang, Q.; Zhang, R.; Du, X. SilicaCoupled Carbon Nanodots: Multicolor Fluorescence Governed by the Surface Structure for Fingerprint Recognition and Wled Devices. New J. Chem. 2021, 45, 11596-11606.

4. Zhan, Y.; Shang, B.; Chen, M.; Wu, L. One-Step Synthesis of Silica-Coated Carbon Dots with Controllable Solid-State Fluorescence for White Light-Emitting Diodes. Small 2019, 15, e1901161.

5. Wu, J.; Tong, J.; Gao, Y.; Wang, A.; Zhang, T.; Tan, H.; Nie, S.; Deng, Z. Efficient and Stable Thin-Film Luminescent Solar Concentrators Enabled by Near-Infrared Emission Perovskite Nanocrystals. Angew. Chem. Int. Ed. 2020, 59, 7738-7742.

6. Zhao, H.; Zhou, Y.; Benetti, D.; Ma, D.; Rosei, F. Perovskite Quantum Dots Integrated in Large-Area Luminescent Solar Concentrators. Nano Energy 2017, 37, 214-223.

7. Bagherzadeh-Khajehmarjan, E.; Nikniazi, A.; Olyaeefar, B.; Ahmadi-Kandjani, S.; Nunzi, J.-M. Bulk Luminescent Solar Concentrators Based on Organic-Inorganic Ch3nh3pbbr3 Perovskite Fluorophores. Sol. Energy Mater. Sol. Cells 2019, 192, 44-51.

8 Zhao, H.; Benetti, D.; Tong, X.; Zhang, H.; Zhou, Y.; Liu, G.; Ma, D.; Sun, S.; Wang, Z. M.; Wang, Y.; Rosei, F. Efficient and Stable Tandem Luminescent Solar Concentrators Based on Carbon Dots and Perovskite Quantum Dots. Nano Energy 2018, 50, 756-765.

9. Zhou, Y.; Benetti, D.; Tong, X.; Jin, L.; Wang, Z. M.; Ma, D.; Zhao, H.; Rosei, F. Colloidal Carbon Dots Based Highly Stable Luminescent Solar Concentrators. Nano Energy 2018, 44, 378-387. 